\title{
On polynomial reduction of problems based on diagonal Latin squares to the exact cover problem
}

\author{
Eduard Vatutin ${ }^{1}$, Natalia Nikitina ${ }^{2}$, Alexey Belyshev ${ }^{3}$ and \\ Maxim Manzyuk ${ }^{3}$ \\ ${ }^{1}$ Southwest State University, 9450 Let Oktyabrya St, Kursk, 305040, Russian Federation \\ ${ }^{2}$ Institute of Applied Mathematical Research, Karelian Research Center RAS, 11 \\ Pushkinskaya St, Petrozavodsk, 185910, Russian Federation \\ ${ }^{3}$ Internet portal BOINC.ru, Russian Federation \\ E-mail: evatutin@rambler.ru, nikitina@krc.karelia.ru, alexey-bell@yandex.ru, \\ hoarfrost@rambler.ru
}

\begin{abstract}
The paper discusses the reduction of problems based on Latin squares to the exact cover problem aiming at its subsequent solution using the dancing links algorithm. The former problems include generation of Latin squares and diagonal Latin squares of a general form/with a given normalization, generation of orthogonal Latin and diagonal Latin squares directly/through the set of transversals, obtaining a set of transversals for a given square, forming a subset of disjoint transversals. For each subproblem, we describe in detail the process of forming the corresponding binary coverage matrices. We show that the use of the proposed approach in comparison with the classical one, i.e. the formation of sets of transversals and their coverages using exhaustive enumeration, allows one to increase the effective processing pace of diagonal Latin squares by $2.5-5.6$ times. The developed software implementations of the algorithms are used in computational experiments as part of the Gerasim@Home volunteer distributed computing project on the BOINC platform
\end{abstract}

\section{Introduction}

One of the known types of combinatorial objects are the Latin Squares (LS) [1, 2], which are square tables of size $N \times N$ cells, where $N$ is the order of the square, filled with elements of some alphabet $U$ (for definiteness in this paper, by integers from 0 to $N-1$ ), so that in each row and each column the elements of the alphabet are not repeated. For Diagonal Latin Squares (DLS), an additional restriction is introduced on the absence of the same values on the main and secondary diagonals. A number of scientific publications study the properties of LS and DLS. A number of open questions from the field of enumerative combinatorics [3] and open mathematical problems are related to them, the most famous of which is the problem of the existence or nonexistence of a triple of mutually orthogonal LS/DLS of order 10. Orthogonal, abbr. OLS/ODLS, are such squares $A=\left(a_{i j}\right)$ and $B=\left(b_{i j}\right)$ for which all ordered pairs $\left(a_{i j}, b_{i j}\right), i, j=\overline{0, N-1}$ are different.

Currently, all ODLS of orders 1-8 and their properties are known up to an isomorphism in

Copyright (C) 2020 for this paper by its authors. Use permitted under Creative Commons License Attribution 4.0 International (CC BY 4.0). 
the corresponding main classes ${ }^{1}$. For higher orders, it is currently not possible to obtain all ODLS due to their large number on the one hand and, as a consequence, huge computational costs on the other hand. Currently, in the projects of volunteer computing RakeSearch [4] and Gerasim@Home [5], the lists of canonical forms (lexicographically minimal representatives within the corresponding main classes) of the ODLS of orders 9 and 10 are being constructed. Moreover, the computations within the RakeSearch project are based on the existence of such ODLS pairs, for some orders [6], in which an orthogonal mate is obtained from the original one by rearranging its rows (a special case of orthogonality of the ESOLS type (Extended SelfOrthogonal Latin Squares) [7], which allows one to raise the processing rate of squares to a value of about 70,000-80,000 DLS/s. The Gerasim@Home project uses a general classical approach based on the Euler-Parker method [8], in which the presence of a general-type ODLS mate for a given square is checked in two stages. They are construction of a set of diagonal transversals and search for subsets of $N$ disjoint transversals in its composition. At the same time, both tasks are actually polynomially reduced to solving the exact cover problem that is NP-complete [9], the solution of which is possible both directly, using the appropriate recursive algorithms, and using the well-known dancing links algorithm (DLX [10,11]). This paper provides meticulous study of the details of this procedure for polynomial reduction of initial particular problems on the basis of LS/DLS to DLX and estimation of speed characteristics of the corresponding software implementations.

\section{DLX algorithm for solving the exact cover problem}

The DLX algorithm for solving the exact cover problem is based on constructing the corresponding binary coverage matrix of size $R$ rows by $C$ columns, followed by finding such subsets of rows whose units exactly (without intersections) cover all columns. To solve problems based on LS/DLS, it is necessary to form various coverage matrices, which is non-trivial and is discussed in detail below.

When forming an $L S$, the rows of the binary matrix correspond to all possible combinations of triples $(i, j, \nu)$, where $i$ is the row number of the formed square, $j$ is the column number, $\nu$ is the value placed in the cell $a_{i j}$, the total number of rows is $N^{3}$. The columns of the binary matrix correspond to three semantic groups of $N^{2}$ elements each:

(i) The $i$-th row $r_{i}$ of the square contains the value of $\nu$ ( $N^{2}$ columns of the binary matrix),

(ii) The $j$-th column $c_{j}$ contains the value of $\nu$ ( $N^{2}$ columns of the binary matrix),

(iii) The cell of a square with coordinates $[i, j]$ is used ( $N^{2}$ columns of a binary matrix).

The binary matrix that corresponds to forming an LS of order 3 is shown in Fig. 1.

When forming a $D L S$, two more groups of columns are added to the matrix considered above:

(i) The main diagonal of the square contains the value $\nu$ ( $N$ columns),

(ii) The secondary diagonal of the square contains the value $\nu$ ( $N$ columns).

The binary matrix that corresponds to forming an LS of order 3 is shown in Fig. 2.

When forming $L S / D L S$ normalized by their first row, by definition, the elements of the first row are specified: $a_{00}=0, a_{01}=1, \ldots, a_{0, N-1}=N-1$, and one cannot set any other values in their places, which corresponds to the special first row and gaps (empty rows without units) in the binary matrix. An example of such a binary matrix is shown in Fig. 3, where blank lines are highlighted in gray. In order to reduce the problem dimension, empty rows can be removed from the matrix.

To search for a set of diagonal transversals in the $L S / D L S$, the rows of the binary matrix correspond to the elements $a_{i j}$ of the square, included in the transversal. The columns of the matrix correspond to the following semantic groups of elements:

1 http://evatutin.narod.ru/evatutin_odls_1_to_8.zip 
(i) Transversal element $a_{i j}$ covers the $i$-th row of the square ( $N$ columns),

(ii) Transversal element $a_{i j}$ covers the $j$-th column of the square ( $N$ columns),

(iii) Transversal element $a_{i j}=\nu$ covers the value of $\nu$ in the set of values used in the transversal ( $N$ columns),

(iv) Element $a_{i j}$ is located on the main diagonal ( 1 column),

(v) Element $a_{i j}$ is located on the secondary diagonal (1 column).

\begin{tabular}{|c|c|c|c|c|c|c|c|c|c|c|c|c|c|c|c|c|c|c|c|c|c|c|c|c|c|c|c|}
\hline & $\begin{array}{l}0 \\
11 \\
: 0\end{array}$ & 픙 & $\begin{array}{l}G r \\
\text { N } \\
\text { II } \\
\text { e }\end{array}$ & II & $\begin{array}{l}11 \\
5\end{array}$ & $\begin{array}{l}r_{i}= \\
\sim \\
\text { II }\end{array}$ & $\begin{array}{l}=v \\
0 \\
\text { II } \\
\approx\end{array}$ & II & $\begin{array}{l}\sim \\
\mathbb{N} \\
\stackrel{N}{11}\end{array}$ & $\begin{array}{l}0 \\
11 \\
0\end{array}$ & $\begin{array}{l}\text { II } \\
\text { \& }\end{array}$ & $\begin{array}{l}\mathrm{Gr} \\
\sim \\
11 \\
8\end{array}$ & $\begin{array}{l}\text { roup } \\
0 \\
11 \\
5\end{array}$ & $\begin{array}{l}0 \text { 2: } \\
\text { i } \\
0\end{array}$ & $\begin{array}{c}c_{i}= \\
\sim \\
11 \\
\vdots\end{array}$ & $\begin{array}{c}=v \\
0 \\
" 1 \\
\text { N }\end{array}$ & & $\begin{array}{l}N \\
\text { II } \\
\text { N }\end{array}$ & षి & & ชี้ & 워 & 3: a & $\frac{\pi}{\pi}$ & ซิ & & สี \\
\hline$a_{00}:=0$ & \begin{tabular}{|l|}
1 \\
\end{tabular} & & & & & & & & & 1 & & & & & & & & & 1 & & & & & & & & \\
\hline$a_{00}:=1$ & & 1 & & & & & & & & & 1 & & & & & & & & 1 & & & & & & & & \\
\hline$a_{00}:=2$ & & & 1 & & & & & & & & & 1 & & & & & & & 1 & & & & & & & & \\
\hline$a_{01}:=0$ & \begin{tabular}{|l|}
1 \\
\end{tabular} & & & & & & & & & & & & 1 & & & & & & & 1 & & & & & & & \\
\hline$a_{01}:=1$ & & 1 & & & & & & & & & & & & 1 & & & & & & 1 & & & & & & & \\
\hline$a_{01}:=2$ & & & 1 & & & & & & & & & & & & 1 & & & & & 1 & & & & & & & \\
\hline$a_{02}:=0$ & \begin{tabular}{|l|}
1 \\
\end{tabular} & & & & & & & & & & & & & & & 1 & & & & & 1 & & & & & & \\
\hline$a_{02}:=1$ & & 1 & & & & & & & & & & & & & & & 1 & & & & 1 & & & & & & \\
\hline$a_{02}:=2$ & & & 1 & & & & & & & & & & & & & & & 1 & & & 1 & & & & & & \\
\hline$a_{10}:=0$ & & & & 1 & & & & & & 1 & & & & & & & & & & & & 1 & & & & & \\
\hline$a_{10}:=1$ & & & & & 1 & & & & & & 1 & & & & & & & & & & & 1 & & & & & \\
\hline$a_{10}:=2$ & & & & & & 1 & & & & & & 1 & & & & & & & & & & 1 & & & & & \\
\hline$a_{11}:=0$ & & & & 1 & & & & & & & & & 1 & & & & & & & & & & 1 & & & & \\
\hline$a_{11}:=1$ & & & & & 1 & & & & & & & & & 1 & & & & & & & & & 1 & & & & \\
\hline$a_{11}:=2$ & & & & & & 1 & & & & & & & & & 1 & & & & & & & & 1 & & & & \\
\hline$a_{12}:=0$ & & & & 1 & & & & & & & & & & & & 1 & & & & & & & & 1 & & & \\
\hline$a_{12}:=1$ & & & & & 1 & & & & & & & & & & & & 1 & & & & & & & 1 & & & \\
\hline$a_{12}:=2$ & & & & & & 1 & & & & & & & & & & & & 1 & & & & & & 1 & & & \\
\hline$a_{20}:=0$ & & & & & & & 1 & & & 1 & & & & & & & & & & & & & & & 1 & & \\
\hline$a_{20}:=1$ & & & & & & & & 1 & & & 1 & & & & & & & & & & & & & & 1 & & \\
\hline$a_{20}:=2$ & & & & & & & & & 1 & & & 1 & & & & & & & & & & & & & 1 & & \\
\hline$a_{21}:=0$ & & & & & & & 1 & & & & & & 1 & & & & & & & & & & & & & 1 & \\
\hline$a_{21}:=1$ & & & & & & & & 1 & & & & & & 1 & & & & & & & & & & & & 1 & \\
\hline$a_{21}:=2$ & & & & & & & & & 1 & & & & & & 1 & & & & & & & & & & & 1 & \\
\hline$a_{22}:=0$ & & & & & & & 1 & & & & & & & & & 1 & & & & & & & & & & & 1 \\
\hline$a_{22}:=1$ & & & & & & & & 1 & & & & & & & & & 1 & & & & & & & & & & 1 \\
\hline$a_{22}:=2$ & & & & & & & & & 1 & & & & & & & & & 1 & & & & & & & & & 1 \\
\hline
\end{tabular}

Figure 1. A binary matrix of size $N^{3} \times 3 N^{2}$ for the formation of an LS of order $N=3$ (hereinafter, the empty elements of the binary matrix correspond to zero values). The lines of one of the possible coverages corresponding to the square $\left(\begin{array}{lll}0 & 1 & 2 \\ 1 & 2 & 0 \\ 2 & 0 & 1\end{array}\right)$ are highlighted in gray.

When forming transversals of a general form (non-diagonal), the last two semantic groups and the corresponding two columns of the matrix should be excluded from consideration. Note that when forming a coverage, the values of groups 1,2, 4, and 5 are general and independent of the processed DLS, while the values of group 3 are determined by the specific values of the elements of the processed DLS, for which a set of transversals is formed. An example of a binary matrix for the formation of a set of diagonal transversals for an LS $\left(\begin{array}{lll}0 & 1 & 2 \\ 1 & 2 & 0 \\ 2 & 0 & 1\end{array}\right)$ is shown in Fig. 4. 
To search for an ODLS mate $B$ for a given DLS A directly (without using transversals), the binary matrix has $N^{3}$ rows corresponding, as in the search for LS/ DLS, to all possible combinations of triples $(i, j, \nu)$. The columns of the matrix correspond to two semantic groups:

(i) The formation of the correct DLS $B\left(3 N^{2}+2 N\right.$ columns $)$,

(ii) The uniqueness of pairs of values $\left(\nu_{1}, \nu_{2}\right), \nu_{1}=a_{i j}, \nu_{2}=b_{i j}\left(N^{2}\right.$ columns by the number of pairs).

Group 1: $r_{i}=v$

Group 2: $c_{i}=v$

Group 3: $a_{i j}$ filled

Groups 4 and 5

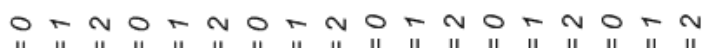

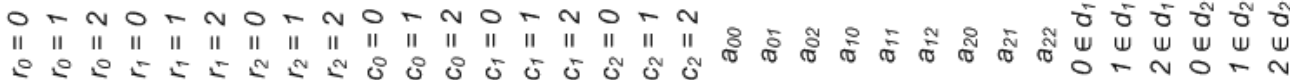

$a_{00}:=0$
$a_{00}:=1$
$a_{00}:=2$
$a_{01}:=0$
$a_{01}:=1$

Figure 2. A binary matrix of size $N^{3} \times\left(3 N^{2}+2 N\right)$ for the formation of DLS of order $N=3$.

To form an $O L S$ from the first semantic group of the matrix, it is necessary to exclude $2 N$ columns corresponding to restrictions on the uniqueness of values on the diagonals.

To form the reduced OLS/ODLS pairs, it is necessary, as already discussed above, to form a special first row of the binary matrix corresponding to the pairs of elements $(0,0),(1,1), \ldots,(N-$ $1, N-1)$ in the first rows of squares of the formed pair, and to remove from the binary matrix the empty lines corresponding to the assignment $\left(a_{0 i}:=\nu_{1}\right) \wedge\left(b_{0 i}:=\nu_{2}\right)$ of values of the fixed elements. An example of a binary matrix corresponding to the formation of the ODLS mate to a given DLS is shown in Fig. 5.

When forming an ODLS from a set of transversals, each row of the binary matrix corresponds to one of the transversals, the columns of the matrix correspond to the elements $a_{i j}$ of the formed orthogonal square that are part of the transversal. An example of a square, its set of transversals and the corresponding binary matrix are shown in Fig. 6.

When forming ODLS pairs directly (without transversals), the corresponding binary matrix 
will have $N^{4}$ rows corresponding to all possible combinations of values $\left(i, j, \nu_{1}, \nu_{2}\right)$, and

$$
\underbrace{\left(3 N^{2}+2 N\right)}_{D L S A}+\underbrace{\left(3 N^{2}+2 N\right)}_{D L S B}+\underbrace{N^{2}}_{\text {pairs }\left(a_{i j}, b_{i j}\right)=\left(\nu_{1}, \nu_{2}\right)}=7 N^{2}+4 N
$$

columns that correspond to the correctness conditions for the pair of squares $A$ and $B$ in conjunction with the uniqueness of the pairs $\left(\nu_{1}, \nu_{2}\right)$.
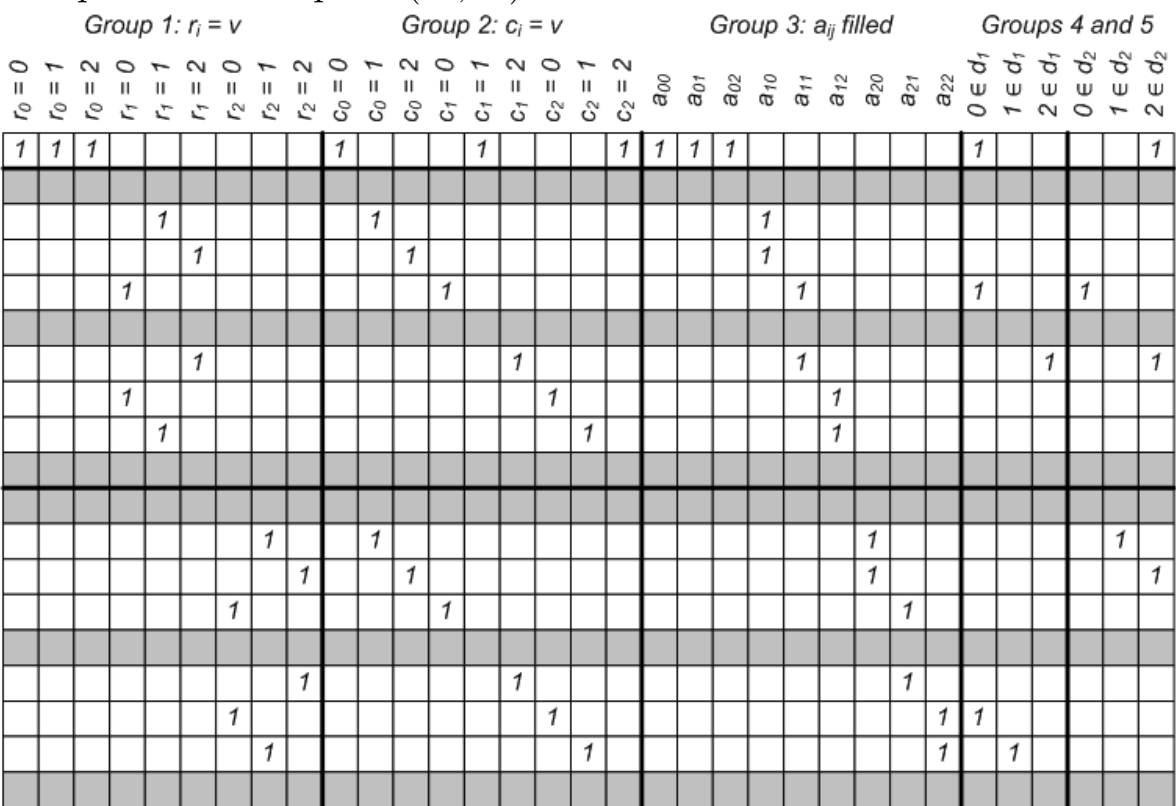

Figure 3. A binary matrix of size $\left(N^{2}(N-1)+1\right) \times\left(3 N^{2}+2 N\right)$ for forming a DLS normalized on the first row of order $N=3$. Empty lines for clarity are left in the matrix, by deleting them the dimension of the problem (matrix size) is reduced up to $\left(N(N-1)^{2}+1\right) \times\left(3 N^{2}+2 N\right)$.

It is easy to notice that the binary matrices given above, corresponding to various problems based on LS/DLS/OLS/ODLS, are significantly sparse, which makes it efficient to use the DLX dancing links algorithm in the search for coverages.

$$
\begin{aligned}
& \text { Group Group Group Groups }
\end{aligned}
$$

\begin{tabular}{|c|c|c|c|c|c|c|c|c|c|c|}
\hline$a_{00}=0$ & 1 & & & & & 1 & & & $1 \mid$ & \\
\hline$a_{01}=1$ & 1 & & & 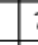 & & & 1 & & & \\
\hline$a_{02}=2$ & 1 & & & & 1 & & & 1 & & 1 \\
\hline$a_{10}=1$ & & 1 & & & & & 1 & & & \\
\hline$a_{11}=2$ & & 1 & & 1 & & & & 1 & & 1 \\
\hline$a_{12}=0$ & & 1 & & & 1 & 1 & & & & \\
\hline$a_{20}=2$ & & & 1 & & & & & 1 & & 1 \\
\hline$a_{21}=0$ & & & 1 & & & 1 & & & & \\
\hline$a_{22}=1$ & & & 1 & & 1 & & & & \begin{tabular}{|l|l|}
1 \\
\end{tabular} & \\
\hline
\end{tabular}

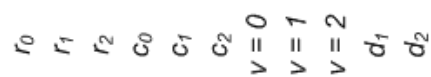

Figure 4. A binary matrix of size $N^{2} \times(3 N+2)$ to form the set of diagonal transversals of the $\operatorname{LS}\left(\begin{array}{lll}0 & 1 & 2 \\ 1 & 2 & 0 \\ 2 & 0 & 1\end{array}\right)$. 


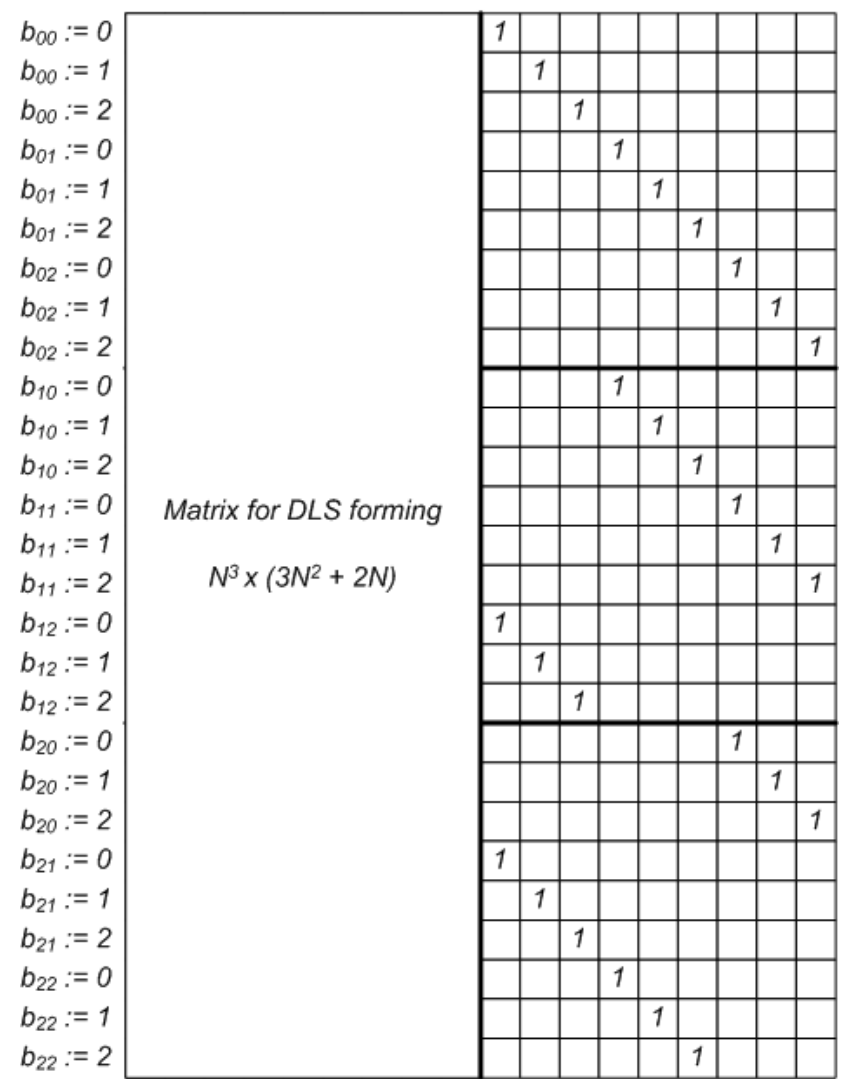

Figure 5. A binary matrix of size $N^{3} \times\left(4 N^{2}+2 N\right)$ for forming the ODLS for the given LS $\left(\begin{array}{lll}0 & 1 & 2 \\ 1 & 2 & 0 \\ 2 & 0 & 1\end{array}\right)$. The values of group 2 depend on the values of elements of square A.

a)
\begin{tabular}{|l|l|l|}
\hline 0 & 1 & 2 \\
\hline 1 & 2 & 0 \\
\hline 2 & 0 & 1 \\
\hline
\end{tabular}

\begin{tabular}{|c|c|c|c|c|c|c|c|c|}
\hline b) & & $t_{1}$ & & & $t_{2}$ & & & $t_{3}$ \\
\hline 0 & 1 & 2 & 0 & 1 & 2 & 0 & 1 & 2 \\
\hline 1 & 2 & 0 & 1 & 2 & 0 & 1 & 2 & 0 \\
\hline 2 & 0 & 1 & 2 & 0 & 1 & 2 & 0 & 1 \\
\hline
\end{tabular}

c) $b_{i j}$

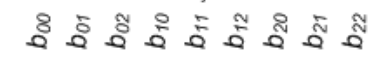

\begin{tabular}{l|l|l|l|l|l|l|l|l|l|}
\cline { 2 - 7 }$t_{1}$ & 1 & & & & 1 & & & & 1 \\
\hline & & 1 & & & & 1 & 1 & & \\
\hline$t_{2}$ & & & & & & \\
\cline { 2 - 7 } & & & 1 & 1 & & & & 1 & \\
\hline
\end{tabular}

Figure 6. A binary matrix of size $T \times N^{2}$, where $T$ is the number of transversals, to form OLS/ODLS of order $N=3$ : initial square (a), set of transversals (b), binary matrix (c).

Tables 1-3 show the sizes of binary matrices for solving the problems considered above for squares of order $N \leq 10$. The size of the coverage matrices polynomially depends from the 
dimension of the problem being solved (from the LS/DLS order $N$ ), and the corresponding algorithms for their formation are quite simple and have an asymptotic polynomial time complexity not exceeding $O\left(N^{3}\right)$.

Table 1. Sizes of binary matrices in the exact cover problem to form LS/DLS depending on the order of squares $N$

\begin{tabular}{lllll}
\hline & Construction of LS & Construction of DLS & $\begin{array}{l}\text { Construction of } \\
\text { normalized LS }\end{array}$ & $\begin{array}{l}\text { Construction of } \\
\text { normalized DLS }\end{array}$ \\
\cline { 2 - 5 } $\mathrm{N}$ & $N^{3} \times 3 N^{2}$ & $N^{3} \times\left(3 N^{2}+2 N\right)$ & $\left(N(N-1)^{2}+1\right) \times 3 N^{2}$ & $\begin{array}{l}\left(N(N-1)^{2}+1\right) \times \\
\times\left(3 N^{2}+2 N\right)\end{array}$ \\
\hline 2 & $8 \times 12$ & $8 \times 16$ & $3 \times 12$ & $3 \times 16$ \\
3 & $27 \times 27$ & $27 \times 33$ & $13 \times 27$ & $13 \times 33$ \\
4 & $64 \times 48$ & $64 \times 56$ & $37 \times 48$ & $37 \times 56$ \\
5 & $125 \times 75$ & $125 \times 85$ & $81 \times 75$ & $81 \times 85$ \\
6 & $216 \times 108$ & $216 \times 120$ & $151 \times 108$ & $151 \times 120$ \\
7 & $343 \times 147$ & $343 \times 161$ & $253 \times 147$ & $253 \times 161$ \\
8 & $512 \times 192$ & $512 \times 208$ & $393 \times 192$ & $393 \times 208$ \\
9 & $729 \times 243$ & $729 \times 261$ & $577 \times 243$ & $577 \times 261$ \\
10 & $1000 \times 300$ & $1000 \times 320$ & $811 \times 300$ & $811 \times 320$ \\
\hline
\end{tabular}

Table 2. Sizes of binary matrices in the exact cover problem to form sets of transversals for LS/DLS depending on the order of squares $N$

\begin{tabular}{lll}
\hline \multirow{2}{*}{$\mathrm{N}$} & $\begin{array}{l}\text { Construction of the set } \\
\text { of transversals of LS/DLS }\end{array}$ & $\begin{array}{l}\text { Construction of the set } \\
\text { of diagonal transversals of LS/DLS }\end{array}$ \\
\cline { 2 - 3 }$N^{2} \times 3 N$ & $N^{2} \times(3 N+2)$ \\
\hline 2 & $4 \times 6$ & $4 \times 8$ \\
4 & $9 \times 9$ & $9 \times 11$ \\
5 & $16 \times 12$ & $16 \times 14$ \\
6 & $25 \times 15$ & $25 \times 17$ \\
7 & $49 \times 18$ & $36 \times 20$ \\
8 & $64 \times 24$ & $49 \times 23$ \\
9 & $81 \times 27$ & $64 \times 26$ \\
10 & $100 \times 30$ & $81 \times 29$ \\
\hline
\end{tabular}

In the early software implementation the search for transversals and covers from them was implemented using the exhaustive search method. In this case, the strategy of early cutting off unpromising solutions (branches and bound method [12]), the variation of the filling order of transversal elements in accordance with the principle of minimum capabilities and the use of bit arithmetic were tested and the processing rate $357 \mathrm{DLS} / \mathrm{s}$ was reached [13]. Reduction of the problem of checking random DLS for the presence of ODLS to the search for the exact covers through transversals in two stages (searching for diagonal transversals, searching for the cover of a square with disjoint diagonal transversals), using the developed DLX implementation, 
allowed us to increase the processing rate to $900 \mathrm{DLS} / \mathrm{s}$ for single-threaded implementation in Delphi on the processor Intel Core i7 4770 (Haswell). In fact, two implementations of the DLX algorithm were developed: one based on a two-dimensional array, the other based on a dynamic list. Array-based implementation is suitable for debugging, but list-based implementation turned out to be faster. In [14], DLX was used to find all orthogonal mates for randomly generated DLS of order 10. The resulting performance was about $899 \mathrm{DLS} / \mathrm{s}$ on 1 CPU core. Note, that in [9] DLX outperformed both SAT approach and backtrack search. Using a similar software implementation in $\mathrm{C}++$ allows one to achieve an effective processing rate of about $7000-8000$ $\mathrm{DLS} / \mathrm{s}$ in the same conditions within the special procedure named canonization (search for symmetrically placed transversals in the LS, putting them in place of the main and secondary diagonals of a square by rearranging its rows and columns [15]). The way to build a faster procedure for checking the DLS for the presence of ODLS is currently unknown.

Table 3. Sizes of binary matrices in the exact cover problem to form OLS/ODLS depending on the order of squares $N$

\begin{tabular}{lllll}
\hline & $\begin{array}{l}\text { Search for ODLS for } \\
\text { a given DLS directly }\end{array}$ & $\begin{array}{l}\text { Search for OLS for } \\
\text { a given LS directly }\end{array}$ & $\begin{array}{l}\text { Search for OLS } \\
\text { using transversals }\end{array}$ & $\begin{array}{l}\text { Search for ODLS } \\
\text { using transversals }\end{array}$ \\
\cline { 2 - 5 } & $N^{3} \times 4 N^{2}+2 N$ & $N^{3} \times 4 N^{2}$ & $T \times N^{2}$ & $T \times N^{2}$ \\
\hline 2 & $8 \times 20$ & $8 \times 16$ & $0 \times 4$ & $0 \times 4$ \\
3 & $27 \times 42$ & $27 \times 36$ & $(3-?) \times 9$ & $0 \times 9$ \\
4 & $64 \times 72$ & $64 \times 64$ & $(0-8) \times 16$ & $4 \times 16$ \\
5 & $125 \times 110$ & $125 \times 100$ & $(3-15) \times 25$ & $(1-5) \times 25$ \\
6 & $216 \times 156$ & $216 \times 144$ & $(0-32) \times 36$ & $(2-6) \times 36$ \\
7 & $343 \times 210$ & $343 \times 196$ & $(3-133) \times 49$ & $(0-27) \times 49$ \\
8 & $512 \times 272$ & $512 \times 256$ & $(0-384) \times 64$ & $(0-120) \times 64$ \\
9 & $729 \times 342$ & $729 \times 324$ & $(68-?) \times 81$ & $? \times 81$ \\
10 & $1000 \times 420$ & $1000 \times 400$ & $(0-5504) \times 100$ & $(?-866) \times 100$ \\
\hline
\end{tabular}

Note: symbol "?" in the table indicates the quantities whose exact value is currently unknown.

\section{Conclusion}

Thus, the problems considered above allow effective polynomial reduction to the exact cover problem and its solution using DLX. The developed software implementations of the algorithms for constructing cover matrices, DLX, and reconstructing solutions from the found covers are currently actively used in the Gerasim@Home volunteer computing project within the BOINC platform [16], and the speed characteristics of the search for ODLS of order 10 are limited by this combination of software implementations of these algorithms. At the moment, their use made it possible to find a list of 9.7 million canonical forms of ODLS, including 24 different types of combinatorial structures (graphs from DLS on the set of binary orthogonality relation) [17]. Most part of them were found via the approach proposed in the paper in combination with different LS generators (for general type DLS, plane symmetric DLS, generalized symmertic DLS, neighborhoods of generalized symmetries of DLS) in the input of the considered Euler-Parker algorithm. In some experiments within the project the search for ODLS with special types of orthogonality can be performed without the use of transversals (for example, for Self-Orthogonal DLS (abbr. SODLS) [18] and Extended Self-Orthogonal DLS (abbr. ESODLS)) and limited by the speed characteristics of the appropriate generators, however, post-processing of the obtained results still requires the use of the Euler-Parker method with DLX inside. Unfortunately, no 
one of the found combinatorial structures includes a triple of pairwise orthogonal DLS or a clique of greater cardinality. Also, these program implementations have been used in solving some enumeration problems that allowed us to discover a set of new numerical series within the Online Encyclopedia of Integer Sequences (OEIS) [19, 20] (A287644, A287645, A287647, A287648, A287651, A287695, A305568, A305569, A305570, A305571, A328873, etc.).

\section{Acknowledgments}

The work was supported by the Russian Foundation of Basic Research, project 18-07-00628. Authors thank citerra [Russia Team] from the internet portal BOINC.ru for his help in the development and implementation of some algorithms and corresponding productive discussions.

\section{References}

[1] Colbourn C J and Dinitz J H 2006 Handbook of Combinatorial Designs, Second Edition (Discrete Mathematics and Its Applications) (Chapman \& Hall/CRC) ISBN 1584885068 URL http://dx.doi.org/10.1201/9781420010541

[2] Keedwell A D and Denes J 2015 Latin Squares and their applications (Elsevier) URL http://dx.doi.org/10.1016/C2014-0-03412-0

[3] Bona M 2015 Handbook of Enumerative Combinatorics (Apple Academic Press Inc.) ISBN 1482220857

[4] 2020 Volunteer computing project RakeSearch Available at https://rake.boincfast.ru/rakesearch/

[5] 2020 Volunteer computing project Gerasim@Home Available at http://gerasim.boinc.ru

[6] Manzyuk M, Nikitina N and Vatutin E 2019 Russian Supercomputing Days (Springer) pp 725-734

[7] 2020 Extended self-orthogonal diagonal latin squares Available at https://oeis.org/A309210

[8] Parker E T 1959 Proc. Natl. Acad. Sci. USA 45 859-862

[9] Karp R M 1972 Complexity of Computer Computations pp 85-103

[10] Knuth D E 2000 Millenial Perspectives in Computer Science 187-214

[11] Knuth D E 2013 The art of computer programming, Volume 4A: combinatorial algorithms (Addison-Wesley Professional) ISBN 0201038048

[12] Land A H and Doig A G 1960 Econometrica 28 497-520 ISSN 00129682

[13] Vatutin E I, Kochemazov S and Zaikin O S 2017 Visual analytic 2017 (Kuzbassvuzizdat) pp 44-49

[14] Kochemazov S, Zaikin O and Semenov A 20172017 40th International Convention on Information and Communication Technology, Electronics and Microelectronics (MIPRO) pp 1166-1171

[15] Brown J W, Cherry F, Most L, Most M, Parker E T and Wallis W D 1992 Lecture notes in pure and applied mathematics 139 43-49

[16] Anderson D P 2019 Journal of Grid Computing 1-24

[17] Vatutin E I, Titov V S, Zaikin O S, Kochemazov S E, Manzuk M O and Nikitina N N 2018 CEUR Workshop Proceedings. Proceedings of the VIII International Conference "Distributed Computing and Gridtechnologies in Science and Education" (GRID 2018) 2267 282-287

[18] Brayton R K, Coppersmith D and Hoffman A J 1974 Bulletin of the American Mathematical Society 80 $116-118$

[19] Sloane N J A 1994 Electr. J. Comb. 1

[20] Sloane N J A 2020 The on-line encyclopedia of integer sequences Available at https://oeis.org 